C2008 IEEE. Personal use of this material is permitted. However, permission to reprint/republish this material for advertising or promotional purposes or for creating new collective works for resale or redistribution to servers or lists, or to reuse any copyrighted component of this work in other works must be obtained from the IEEE. 


\title{
Optimum Design of Discrete-Time Differentiators via Semi-Infinite Programming Approach
}

\author{
Charlotte Yuk-Fan Ho, Bingo Wing-Kuen Ling, Yan-Qun Liu, Peter Kwong-Shun Tam, Member, IEEE, \\ and Kok-Lay Teo, Senior Member, IEEE
}

\begin{abstract}
In this paper, a general optimum full-band, highorder discrete-time differentiator design problem is formulated as a peak-constrained least squares optimization problem. That is, the objective of the optimization problem is to minimize the total weighted square error of the magnitude response subject to the peak constraint of the weighted error function. This problem formulation provides great flexibility for the tradeoff between the ripple energy and the ripple magnitude of the discrete-time differentiator. The optimization problem is actually a semi-infinite programming problem. Our recently developed dual parameterization algorithm is applied to solve the problem. The main advantages of employing the dual parameterization algorithm to solve the problem are as follows: 1) the guarantee of the convergence of the algorithm and 2) the obtained solution being the global optimal solution that satisfies the corresponding continuous constraints. Moreover, the computational cost of the algorithm is lower than that of algorithms that are implementing the semidefinite programming approach.
\end{abstract}

Index Terms-Discrete-time differentiators, dual parameterization algorithm, eigen approach, peak-constrained least squares approach, Remez approach, semidefinite programming approach, semi-infinite programming.

\section{INTRODUCTION}

D ISCRETE-TIME differentiators have many important applications in physics and engineering [1]. In particular, they are used to obtain a set of data relating to the rate of change of some physical quantities, such as the estimation of heating rates from temperature data, net flow rates of fluid

Manuscript received July 21, 2005; revised February 12, 2008. This work was supported in part by Research Grant G-YD26 from The Hong Kong Polytechnic University; in part by the Centre for Multimedia Signal Processing, The Hong Kong Polytechnic University; in part by the Central Research Grant Council, Grant PolyU 5105 $\backslash 01 \mathrm{E}$ from the Research Grants Council of Hong Kong; and in part by a Research Grant from the Australian Research Council.

C. Y.-F. Ho is with School of Mathematical Sciences, Queen Mary College, University of London, E1 4NS London, U.K. (e-mail: c.ho@qmul.ac.uk).

B. W.-K. Ling is with the Department of Electronic Engineering, Division of Engineering, King's College London, WC2R 2LS London, U.K. (e-mail: wingkuen.ling@kcl.ac.uk).

Y.-Q. Liu is with the Department of Mathematics and Statistics, Royal Melbourne Institute of Technology University, Melbourne, Vic. 3001, Australia (e-mail: yanqun.liu@rmit.edu.au).

P. K.-S. Tam is with the Department of Electronic and Information Engineering, The Hong Kong Polytechnic University, Kowloon, Hong Kong (e-mail: enptam@polyu.edu.hk).

K.-L. Teo is with the Department of Mathematics and Statistics, Curtin University of Technology, Perth, W.A. 6845, Australia (e-mail: K.L.Teo@ curtin.edu.au)

Color versions of one or more of the figures in this paper are available online at http://ieeexplore.ieee.org.

Digital Object Identifier 10.1109/TIM.2008.922090 from measurements of the volume level, velocity from position data, etc.

Two common methods for the design of discrete-time differentiators are based on the eigen approach [2] and the Remez algorithm approach [3]. However, the eigen approach does not guarantee the obtained solution satisfying the required specifications. On the other hand, the Remez algorithm approach would result in a discrete-time differentiator with large ripple energy. To address this problem, the discrete-time differentiator design problem is formulated as a peak constraint least squares optimization problem. That is, the total weighted square error of the magnitude response is minimized subject to the peak constraint of the weighted error function [4]-[6]. Although this problem formulation provides great flexibility for the tradeoff between the ripple energy and the ripple magnitude of the discrete-time differentiator, this optimization problem is actually a semi-infinite programming problem. The common method for solving semi-infinite programming problems is via the semidefinite programming approach [4]. That is, the continuous constraints are discretized into a finite number of discrete constraints. However, this approach does not guarantee that the continuous constraints are satisfied among the discretization points. Although the deviation between the continuous constraints and the discrete constraints can be reduced by increasing the number of discretization points, the exact number of discretization points that are required for the optimization problem is unknown, and the increase in the number of discrete constraints will result in the increase in the computational complexity. Although a new primal quadratic programming approach was proposed to solve the problem [5], the convergence of the algorithm is not guaranteed. In this paper, the dual parameterization algorithm is employed to solve the problem [6]. The semi-infinite programming problem is reduced to a sequence of approximating subproblems followed by a nonlinear finite programming problem. Each of the approximating subproblems can be readily solved by quadratic programming. The global solution of the finite nonlinear program can then be obtained from the approximated solution. If the feasible set is nonempty, then an exact optimal solution is guaranteed. Also, the convergence of the algorithm is proved. Moreover, since the total number of finite constraints in the approximating subproblems is smaller than that of the corresponding semidefinite programming problems, the computational complexity is low.

The outline of this paper is as follows. In Section II, the optimum discrete-time differentiator design problem is formulated as a semi-infinite programming problem. The dual parameterization algorithm is summarized in Section III. The computer 
numerical simulation results are presented in Section IV. Last, a conclusion is drawn in Section V.

\section{Problem Formulation}

Let $h(n)$ be the impulse response of the discrete-time differentiator. When $N$ is odd, we assume that

$$
\left\{\begin{array}{l}
h(k)=-h(N-1-k), \quad k=0,1,2, \ldots, \frac{N-3}{2} \\
h\left(\frac{N-1}{2}\right)=0
\end{array}\right.
$$

When $N$ is even, we assume that

$$
h(k)=-h(N-1-k) \quad \text { for } \quad k=0,1,2, \ldots, \frac{N}{2}-1 .
$$

Define

$$
\begin{aligned}
\mathbf{x} & \equiv \begin{cases}{\left[a_{1}, a_{2}, \ldots, a_{\frac{N-1}{2}}\right]^{T},} & N \text { is odd } \\
{\left[a_{1}, a_{2}, \ldots, a_{\frac{N}{2}}\right]^{T},} & N \text { is even }\end{cases} \\
\boldsymbol{\eta}(\omega) & \equiv \begin{cases}{\left[\sin \omega, \sin 2 \omega, \ldots, \sin \left(\left(\frac{N-1}{2}\right) \omega\right)\right]^{T},} & N \text { is odd } \\
{\left[\sin \frac{\omega}{2}, \sin \frac{3 \omega}{2}, \ldots, \sin \left(\left(\frac{N-1}{2}\right) \omega\right)\right]^{T},} & N \text { is even }\end{cases} \\
H_{0}(\omega) & \equiv(\boldsymbol{\eta}(\omega))^{T} \mathbf{x}
\end{aligned}
$$

in which

$$
a_{n} \equiv \begin{cases}2 h\left(\frac{N-1}{2}-n\right), & N \text { is odd and } n=1,2, \ldots, \frac{N-1}{2} \\ 2 h\left(\frac{N}{2}-n\right), & N \text { is even and } n=1,2, \ldots, \frac{N}{2} .\end{cases}
$$

Then, the frequency response of the discrete-time differentiator can be expressed as

$$
H(\omega)=j e^{-j \omega\left(\frac{N-1}{2}\right)} H_{0}(\omega)
$$

where $j \equiv \sqrt{-1}$.

Define $B_{d} \equiv[(d / 2)-\pi, \pi-(d / 2)]$, where $d$ is the width of the transition band. Then, the total weighted square error of the magnitude response of the discrete-time differentiator can be represented by

$$
J(\mathbf{x}) \equiv \int_{B_{d}} W(\omega)\left|H_{0}(\omega)-D(\omega)\right|^{2} d \omega=\frac{1}{2} \mathbf{x}^{T} \mathbf{Q} \mathbf{x}+\mathbf{b}^{T} \mathbf{x}+p
$$

where $D(\omega)$ is the desired magnitude response, $W(\omega)$ is the weighted function with $W(\omega)>0$ for $\omega \in B_{d}$, and

$$
\begin{aligned}
\mathbf{Q} & =2 \int_{B_{d}} W(\omega) \boldsymbol{\eta}(\omega)(\boldsymbol{\eta}(\omega))^{T} d \omega \\
\mathbf{b} & =-2 \int_{B_{d}} W(\omega) D(\omega) \boldsymbol{\eta}(\omega) d \omega \\
p & =\int_{B_{d}} W(\omega)(D(\omega))^{2} d \omega .
\end{aligned}
$$

It can be easily checked that matrix $\mathbf{Q}$ is positive definite. To specify the constraints, let $\delta$ be the peak constraint of the weighted error function. Then, the constraint can be expressed as follows:

$$
W(\omega)\left|H_{0}(\omega)-D(\omega)\right| \leq \delta, \quad \text { for } \quad \omega \in B_{d}
$$

which implies that

$$
\mathbf{A}(\omega) \mathbf{x} \leq \mathbf{c}(\omega), \quad \text { for } \quad \omega \in B_{d}
$$

where

$$
\begin{aligned}
\mathbf{A}(\omega) & =W(\omega)[\boldsymbol{\eta}(\omega),-\boldsymbol{\eta}(\omega)]^{T}, \quad \text { for } \quad \omega \in B_{d} \\
\mathbf{c}(\omega) & =[D(\omega) W(\omega)+\delta, \delta-D(\omega) W(\omega)]^{T}, \quad \text { for } \quad \omega \in B_{d} .
\end{aligned}
$$

Clearly, $\mathbf{A}(\omega)$ and $\mathbf{c}(\omega)$ are continuously differentiable with respect to $\omega \in B_{d}$. Consequently, the optimum discrete-time differentiator design problem can be formulated as the following semi-infinite programming problem:

Problem $(\mathbf{P})$

$$
\begin{aligned}
\min _{\mathbf{x}} J(\mathbf{x}) & =\frac{1}{2} \mathbf{x}^{T} \mathbf{Q} \mathbf{x}+\mathbf{b}^{T} \mathbf{x}+p \\
\text { subject to } \mathbf{g}(\mathbf{x}, \omega) & =\mathbf{A}(\omega) \mathbf{x}-\mathbf{c}(\omega) \leq \mathbf{0}, \text { for } \omega \in B_{d} .
\end{aligned}
$$

\section{Dual Parameterization Algorithm}

The above problem can be solved using the dual parameterization algorithm [6]. We first consider Dorn's dual of problem $(\mathbf{P})$ as follows:

Problem $(\mathbf{D})$

$$
\begin{array}{ll}
\min _{(\mathbf{x}, \boldsymbol{\Lambda})} & L(\mathbf{x}, \boldsymbol{\Lambda}) \\
\text { subject to } & \mathbf{Q x}+\mathbf{b}+\mathbf{A}^{*} \boldsymbol{\Lambda}=\mathbf{0}
\end{array}
$$$$
\Lambda \geq \mathbf{0}
$$

where

$$
L(\mathbf{x}, \boldsymbol{\Lambda})=\frac{1}{2} \mathbf{x}^{T} \mathbf{Q} \mathbf{x}+\int_{B_{d}} \mathbf{c}(\omega) d \mathbf{\Lambda} .
$$

A is the operator from $\Re^{N^{\prime}}$ to $C\left(B_{d}, \Re^{N^{\prime}}\right)$, which is defined by $\mathbf{A}(\omega)$ according to $(\mathbf{A x})(\omega)=\mathbf{A}(\omega) \mathbf{x}$ for $\omega \in B_{d}$, where $\mathbf{A}^{*}$ is the dual operator of $\mathbf{A}, N^{\prime}$ is the length of the vector $\mathbf{x}$, and $C\left(B_{d}, \Re^{N^{\prime}}\right)$ is the Banach space of all continuous real functions on $B_{d}$.

Assume that the Slater's qualification holds, that is, there exists $\mathbf{x}_{0} \in \Re^{N^{\prime}}$ satisfying $\mathbf{g}\left(\mathbf{x}_{0}, \omega\right)<\mathbf{0} \forall \omega \in B_{d}$. Since 1) $J$ and $\mathbf{g}(\mathbf{x}, \omega)$ are convex in $\left.\mathbf{x}, \forall \omega \in B_{d} ; 2\right) J$ is differentiable on $\Re^{N^{\prime}}$; and 3) $\mathbf{g}(\mathbf{x}, \omega)$ is continuous in $\omega, \forall \mathbf{x} \in \Re^{N^{\prime}}$, and 
continuously differentiable in $\mathrm{x}$ on $\Re^{N^{\prime}} \times B_{d}$, the strong duality theorem holds. That is, if the minimum of primal problem $(\mathbf{P})$ is achieved by some $\mathbf{x}^{*} \in \Re^{N^{\prime}}$, then there exists a solution $\Lambda^{*}$ of dual problem $(\mathbf{D})$ such that

$$
J\left(\mathbf{x}^{*}\right)=\min _{\mathbf{x}} L\left(\mathbf{x}, \boldsymbol{\Lambda}^{*}\right)
$$

subject to $\quad \int_{B_{d}}\left(\mathbf{A}(\omega) \mathbf{x}^{*}-\mathbf{c}(\omega)\right) d \mathbf{\Lambda}^{*}=\mathbf{0}$

$$
\Lambda^{*} \geq \mathbf{0}
$$

Since $\mathbf{g}(\mathbf{x}, \omega)$ is continuously Fréchet differentiable, the Karush-Kuhn-Tucker (KKT) conditions for problem $(\mathbf{P})$ are also satisfied. That is, the minimum of problem $(\mathbf{P})$ can be achieved at $\mathbf{x}^{*} \in \Re^{N^{\prime}}$ if and only if $\mathbf{x}^{*}$ is feasible, and there exists $\Lambda^{*}$ such that

$$
\begin{aligned}
& \mathbf{Q x}^{*}+\mathbf{b}+\mathbf{A}^{*} \mathbf{\Lambda}^{*}=\mathbf{0} \\
& \int_{B_{d}}\left(\mathbf{A}(\omega) \mathbf{x}^{*}-\mathbf{c}(\omega)\right) d \mathbf{\Lambda}^{*}(\omega)=\mathbf{0} \\
& \boldsymbol{\Lambda}^{*} \geq \mathbf{0} .
\end{aligned}
$$

In general, the multiplier $\Lambda^{*}$ satisfying the KKT conditions is not unique. However, as we assume that the Slater constraint qualification is satisfied, the optimal solution of primal problem $(\mathbf{P})$ is achieved at $\mathbf{x}^{*} \in \Re^{N^{\prime}}$. Therefore, the set of multipliers satisfying the KKT conditions of problem $(\mathbf{P})$ will necessarily include a measure with finite support at no more than $N^{\prime}$ points unless it is empty. This can be proved by Carathéodory's theorem. Hence, there exists a solution pair $\left(\mathbf{x}^{*}, \boldsymbol{\Lambda}^{*}\right)$ of dual problem $(\mathbf{D})$, where the measure $\boldsymbol{\Lambda}^{*}$ has finite support of no more than $N^{\prime}$ points.

Dual semi-infinite problem (D) can be reduced to a finitedimensional optimization problem (PD), which is called the parameterized dual of problem $(\mathbf{P})$, as follows:

\section{Problem $(\mathbf{P D})$}

$$
\begin{array}{ll}
\min _{(\mathbf{x}, \mathbf{t}, \boldsymbol{\lambda})} & L_{k}(\mathbf{x}, \mathbf{t}, \boldsymbol{\lambda}) \\
\text { subject to } & \boldsymbol{\lambda}_{i} \geq \mathbf{0}, \quad i=1,2, \ldots, N^{\prime} \\
& \omega_{i} \in B_{d}, \quad i=1,2, \ldots, k \leq N^{\prime}
\end{array}
$$

where the integer $k$ is the parameterization number, $\mathbf{t}=$ $\left[\omega_{1}, \omega_{2}, \ldots, \omega_{k}\right]^{T}$, and $\boldsymbol{\lambda}=\left[\boldsymbol{\lambda}_{1}, \boldsymbol{\lambda}_{2}, \ldots, \boldsymbol{\lambda}_{k}\right]$, in which $\boldsymbol{\lambda}_{i}=$ $\left[\lambda_{i, 1}, \lambda_{i, 2}, \ldots, \lambda_{i, m}\right]^{T} \in \Re^{m}$, and $m$ is the number of rows in matrix $\mathbf{A}$. The cost function $L_{k}(\mathbf{x}, \mathbf{t}, \boldsymbol{\lambda})$ is given by

$$
L_{k}(\mathbf{x}, \mathbf{t}, \boldsymbol{\lambda})=\frac{1}{2} \mathbf{x}^{T} \mathbf{Q} \mathbf{x}+\sum_{i=1}^{k} \mathbf{c}^{T}\left(\omega_{i}\right) \boldsymbol{\lambda}_{i} .
$$

According to the dual parameterization theory, once a solution $\left(\mathbf{x}^{*}, \mathbf{t}^{*}, \boldsymbol{\lambda}^{*}\right)$ is obtained from solving problem $(\mathbf{P D})$, the optimal solution of primal problem $(\mathbf{P})$ will also be $\mathbf{x}^{*}$. To state the algorithm for solving problem $(\mathbf{P})$, denote the problem ob- tained from problem $(\mathbf{P D})$ by fixing $\mathbf{t}$ as problem $(\mathbf{P D}(\mathbf{t}))$. It can be easily shown that problem $(\mathbf{P D}(\mathbf{t}))$ is the dual problem of problem $(\mathbf{P}(\mathbf{t}))$ for fixed $\omega_{i} \in B_{d}, i=1,2, \ldots, k$, i.e.,

$\operatorname{Problem}(\mathbf{P}(\mathbf{t}))$

$$
\begin{array}{ll}
\min _{\mathbf{x}} & J(\mathbf{x})=\frac{1}{2} \mathbf{x}^{T} \mathbf{Q} \mathbf{x}+\mathbf{b}^{T} \mathbf{x}+p \\
\text { subject to } & \mathbf{g}\left(\mathbf{x}, \omega_{i}\right) \leq \mathbf{0}, \text { for } i=1,2, \ldots, k .
\end{array}
$$

Hence, we have the following theorem:

Theorem 1: Consider problems $(\mathbf{P}),(\mathbf{P}(\mathbf{t}))$, and $(\mathbf{P D})$. The following statements hold.

1) Let $\overline{\mathbf{x}}$ be an optimum solution of problem $(\mathbf{P}(\mathbf{t}))$. If $\overline{\mathbf{x}}$ satisfies the infinite constraint (16b), then $\overline{\mathbf{x}}$ is the optimal solution of primal problem $(\mathbf{P})$.

2) Let $\mathbf{v}_{k}$ be the optimal value of problem $(\mathbf{P D})$ with parameterization number $k$. Then, sequence $\left\{\mathbf{v}_{k}\right\}$ is decreasing, and there exists $k^{*}$ such that $\mathbf{v}_{k^{*}}=\mathbf{v}_{k}$ for all $k \geq k^{*}$. Furthermore, if $k^{*} \geq 1$, then $\mathbf{v}_{k^{*}-1}>\mathbf{v}_{k^{*}}$.

3) The $k^{*}$ in (2) is the minimum integer such that for $k \geq$ $k^{*}$, a global solution of finite problem (PD) provides the solution for primal problem $(\mathbf{P})$ in the sense that if $\left(\mathbf{x}^{*}, \mathbf{t}^{*}, \boldsymbol{\lambda}^{*}\right)$ is a global optimizer of problem $(\mathbf{P D})$, then $\mathbf{x}^{*}$ is the global optimizer of primal problem $(\mathbf{P})$.

4) If $0 \leq k<k^{*}$, then $\mathbf{v}_{k}>\mathbf{v}_{k+1}$.

The proof of Theorem 1 can be found in [6]. The number $k^{*}$ in Theorem 1 is called the minimum parameterization number. If the optimal primal solution is an interior point of the feasible region, then $k^{*}=0$.

Let $\left\{k_{i}\right\}$ be the given sequence of the parameterization numbers satisfying $k_{i} \leq k_{i+1}$. For each $i$, let $\Omega_{i}=\left\{\omega_{j}^{i}\right.$ : $\left.j=1,2, \ldots, k_{i}\right\}$ be a given subset of $B_{d}$, and let $\mathbf{t}^{i}=$ $\left[\begin{array}{llll}\omega_{0}^{i} & \omega_{1}^{i} & \cdots & \omega_{k_{i}}^{i}\end{array}\right]^{T}$. Define the density distance between $\Omega_{i}$ and $B_{d}$ as

$$
d\left(\Omega_{i}, B_{d}\right) \equiv \max _{\omega \in B_{d}} \min _{1 \leq j \leq l_{i}}\left|\omega-\omega_{j}^{i}\right| .
$$

We then have the following theorem.

Theorem 2: Let $\left\{\mathbf{t}^{i}\right\}$ be the sequence given as above. Suppose that $\left(\overline{\mathbf{x}}^{i}, \bar{\lambda}^{i}\right)$ is a solution of problem $\left(\operatorname{PD}\left(\mathbf{t}^{i}\right)\right)$. If $d\left(\Omega_{i}, B_{d}\right) \rightarrow 0$ as $i \rightarrow+\infty$, then the following holds.

1) $\left\{\overline{\mathbf{x}}^{i}\right\}$ converges to the solution of primal problem $(\mathbf{P})$.

2) $\mathbf{v}\left(\mathbf{P D}\left(\mathbf{t}^{i}\right)\right) \rightarrow \mathbf{v}(\mathbf{D})$, where $\mathbf{v}(\mathbf{S})$ denotes the optimal value of a given problem $(\mathbf{S})$.

The proof of Theorem 2 can be found in [6].

We finally obtain the following optimization algorithm.

\section{Algorithm}

Step 0 (initialization): Select a small number $\varepsilon>0$. Choose a sequence of index sets $\Omega_{i}$. Set $i=1$.

Step 1 (compute a local optimum): Solve finite problem $\left(\mathbf{P D}\left(\mathbf{t}^{i}\right)\right)$. Denote the local optimal solution as $\left(\mathbf{x}^{i}, \boldsymbol{\lambda}^{i}\right)$.

Step 2 (test the improvement of the objective): If $i \geq 2$ and $\left|\mathbf{v}\left(\mathbf{P D}\left(\mathbf{t}^{i}\right)\right)-\mathbf{v}(\mathbf{D})\right|<\varepsilon$, then go to step 3; else $i=i+1$ and go to step 1 . 
Step 3 (compute the global optimum): Implement a local search for finite dual problem (PD) with $k=k_{i}$. The solution is denoted as $\left(\mathbf{x}^{*}, \mathbf{t}^{*}, \boldsymbol{\lambda}^{*}\right)$, and $\mathbf{x}^{*}$ is taken as the optimizer of problem $(\mathbf{P})$.

\section{Computer Numerical Simulation Results}

To demonstrate the applicability of our proposed algorithm, a full-band high-order discrete-time differentiator is preferred. However, the magnitude response of the discrete-time differentiator would rise very fast if its order is high. Hence, it requires many filter coefficients for the implementation. To trade off between these two factors, a full-band, fifth-order, discrete-time differentiator is illustrated in this paper. That is, $D(\omega)=\omega^{5} \forall \omega \in B_{d}$. To design a full-band, fifth-order, discrete-time differentiator, a small ripple magnitude and a small transition bandwidth of the differentiator are usually preferred. However, it requires many filter coefficients for the implementation. To trade off among these factors, $N=32, \delta=$ $0.0064 \times \pi^{5}$, and $d=0.06 \pi$ are chosen as the specifications. To demonstrate the performance of the full-band, fifth-order differentiator, the effect of the weighted function should be removed, and a uniform weighted function is employed, i.e., $W(\omega)=1 \forall \omega \in B_{d}$. In our proposed dual parameterization algorithm, a small value of $\varepsilon$ is usually preferred. However, a too-small value of $\varepsilon$ would increase the number of iterations, and therefore, the computational complexity is increased. To trade off between these two factors, $\varepsilon=1 \times 10^{-6}$ is chosen. A large number of discrete frequencies in the index sets are usually preferred. However, too many discrete frequencies would increase the computational complexity. Since the number of extrema of the magnitude response of the full-band, fifth-order differentiator that is designed via the Remez algorithm is equal to $N+2$, the number of discrete frequencies in the first index set is $N+2$. For simplicity, a uniform sampling scheme is employed. Therefore, the first index set is initialized as $\Omega_{1}=\left\{\omega_{k}: \omega_{k}=(d / 2)-\pi+k((2 \pi-d) /(N+1))\right.$ for $k=$ $0,1, \ldots, N+1\}$. The other index sets are constructed based on the previous index set by adding all violated index points of a refined set of grid points to the previous index set while dropping all the unnecessary points from $\Omega_{i-1}$ for $i>1$.

Our computer numerical simulation results are compared to those designed based on the eigen approach [2], the Remez approach [3], and the semidefinite programming approach [4]. These approaches are compared because they are the most common approaches for the design of full-band, high-order differentiators. The magnitude response of the full-band, fifthorder differentiators that are designed via various approaches is shown in Fig. 1, whereas the corresponding weighted error functions are shown in Fig. 2. We can see from Fig. 2 that the maximum ripple magnitude of the full-band, fifth-order differentiator that is designed via the eigen approach is very large that it fails to satisfy the specification. Although the fullband, fifth-order differentiator that is designed via the Remez approach achieves the smallest ripple magnitude among these approaches, the total weighted square error is the largest among these approaches. The full-band, fifth-order differentiator that is designed via the semidefinite programming approach also fails

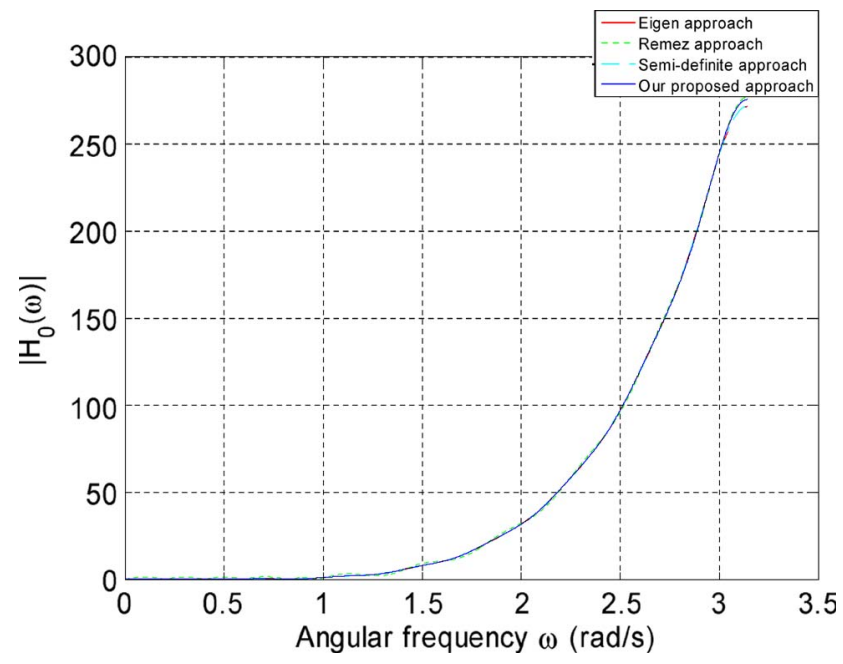

Fig. 1. Magnitude response of the full-band, fifth-order differentiators.

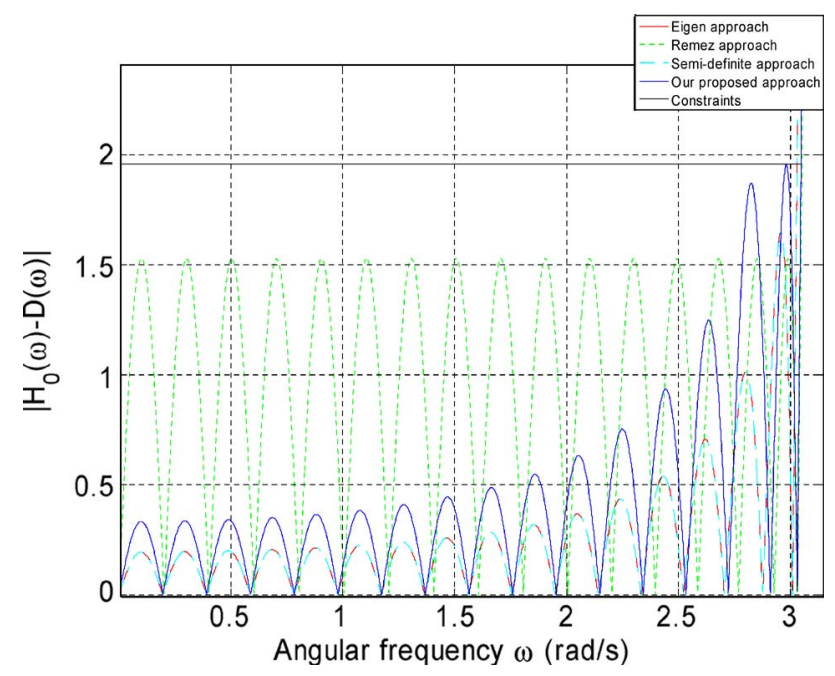

Fig. 2. Weighted error function of the full-band, fifth-order differentiators.

the specification because the number of discrete frequencies is not large enough. On the other hand, the full-band, fifthorder differentiator that is designed via the peak constraint least squares approach satisfies the required specification and minimizes the total weighted square error.

It is difficult to have a fair comparison between the computational complexity of our proposed method and those of other existing methods because almost none of them solve the design problem via the semi-infinite programming approach with the guarantee of the convergence of the algorithms. In our proposed method, it is found that the algorithm terminated after three iterations, and the number of discrete frequencies in the last index set is 1345 . If the design problem is formulated via the semidefinite programming approach with the same number of discrete frequencies, it is shown in Fig. 2 that the solution obtained does not satisfy the specification. Hence, more discrete frequencies are required for the semidefinite programming approach. It implies that the computational complexity of the semidefinite programming approach is much higher than our proposed algorithm [4]. 


\section{CONCLusion}

The main contribution of this paper is the formulation of the optimum discrete-time differentiator design problem as a peakconstrained least squares optimization problem. The problem formulation can be also applied to nonfull-band, arbitraryorder, discrete-time differentiator design problems. The formulated problem is a semi-infinite programming problem, and our proposed dual parameterization algorithm is employed to solve the problem. The main advantages of our proposed algorithm are as follows: 1) the guarantee of the solutions converging to the optimum one that satisfies the continuous constraints if the solution exists; and 2) low computational complexity because the semi-infinite programming problem is transformed to a finite-dimensional optimization problem, and just a few active points are sufficient to give enough information for searching the optimal solution.

\section{REFERENCES}

[1] S. L. Lin and S. Mourad, "On-chip rise-time measurement," IEEE Trans. Instrum. Meas., vol. 53, no. 6, pp. 1510-1516, Dec. 2004.

[2] S.-C. Pei and J.-J. Shyu, "Eigenfilter design of higher-order digital differentiators," IEEE Trans. Acoust., Speech, Signal Process., vol. 37, no. 4, pp. 505-511, Apr. 1989.

[3] E. Z. Psarakis and G. V. Moustakides, "A robust initialization scheme for the Remez exchange algorithm," IEEE Signal Process. Lett., vol. 10, no. 1, pp. 1-3, Jan. 2003.

[4] W.-S. Lu, "A unified approach for the design of 2-D digital filters via semidefinite programming," IEEE Trans. Circuits Syst. I, Fundam. Theory Appl., vol. 49, no. 6, pp. 814-826, Jun. 2002.

[5] J. W. Adams, P. Kruethong, R. Hashemi, J. L. Sullivan, and D. Gleeson, "New quadratic programming algorithms for designing FIR digital filters," in Proc. 27th Asilomar Conf. Signals, Syst. Comput., Nov. 1-3, 1993, vol. 2, pp. $1206-1210$.

[6] Y. Liu, C. H. Tseng, and K. L. Teo, "A unified quadratic semi-infinite programming approach to time and frequency domain constrained digital filter design," Commun. Inf. Syst., vol. 2, no. 4, pp. 399-410, Dec. 2002.

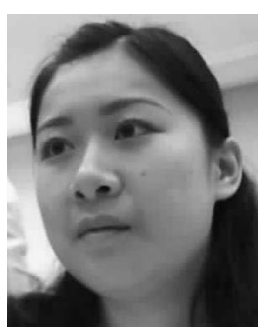

Charlotte Yuk-Fan Ho received the B.Eng. (Hons.) degree from The Hong Kong University of Science and Technology, Kowloon, Hong Kong, in 2000 and the M.Phil. degree from The Hong Kong Polytechnic University, Kowloon, in 2003. She is currently working toward the Ph.D. degree at Queen Mary College, University of London, London, U.K.

Her research interests include investigations of applications of continuous constrained optimizations, symbolic dynamics, filter banks and wavelets, as well as fuzzy and impulsive control theory.

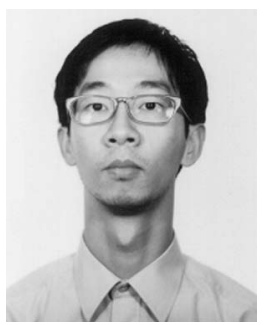

Bingo Wing-Kuen Ling received the B.Eng. (Hons.) and M.Phil. degrees from The Hong Kong University of Science and Technology, Kowloon, Hong Kong, in 1997 and 2000, respectively, and the Ph.D. degree from The Hong Kong Polytechnic University, Kowloon, in 2003.

In 2004, he joined King's College London, London, U.K., as a Lecturer. He has served as an organizer of a special session of the 2008 International Symposium on Communication Systems, Networks, and Digital Signal Processing. He has also served as a Guest Editor of a special session on nonlinear circuits and systems in the Journal of Circuits, Systems and Signal Processing. He is the author of the textbook Nonlinear Digital Filters: Analysis and Applications and an editor of the book Control of Chaos in Nonlinear Circuits and Systems. His research interests include investigations of applications of continuous constrained optimizations, symbolic dynamics, filter banks and wavelets, as well as fuzzy and impulsive control theory.

Mr. Ling has served as a technical committee member of several IEEE international conferences.

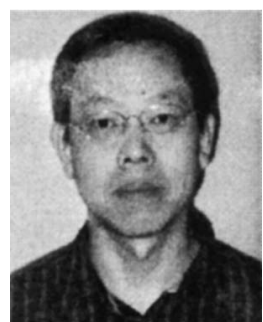

Yan-Qun Liu received the Ph.D. degree in mathematics from The University of Western Australia, Perth, Australia, in 1997.

From 1997 to 1999, he was a Research Associate with School of Mathematics and Statistics, Curtin University of Technology, Perth. From 2000 to 2003, he was a Research Fellow with the Department of Applied Mathematics and, from 2003 to 2004, a Senior Research Fellow with the Department of Electronic and Information Engineering, both at The Hong Kong Polytechnic University, Kowloon, Hong Kong. He is currently a Lecturer with the Department of Mathematics and Statistics, Royal Melbourne Institute of Technology University, Melbourne, Australia. His research interests include optimization, optimal control, and signal processing.

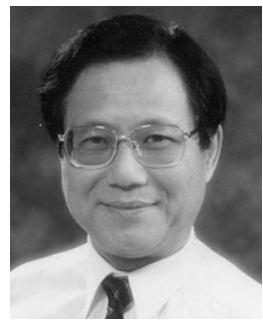

Peter Kwong-Shun Tam (S'74-M'76) received the B.E., M.E., and Ph.D. degrees from The University of Newcastle, Newcastle, Australia, in 1971, 1973, and 1976, respectively, all in electrical engineering.

From 1967 to 1980, he held a number of industrial and academic positions in Australia. In 1980, he joined, as a Senior Lecturer, The Hong Kong Polytechnic University, Kowloon, Hong Kong, where he is currently an Associate Professor with the Department of Electronic and Information Engineering. He has participated in the organization of a number of symposiums and conferences. His research interests include signal processing, automatic control, fuzzy systems, and neural networks.

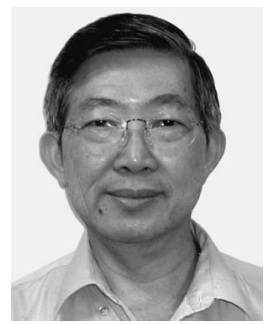

Kok-Lay Teo (M'74-SM'87) received the B.Sc. degree in telecommunications engineering from Ngee Ann Technical College, Singapore, in 1969 and the M.A.Sc. and Ph.D. degrees in electrical engineering from the University of Ottawa, Ottawa, ON, Canada, in 1971 and 1974, respectively.

From 1974 to 1985, he was with the Department of Applied Mathematics, The University of New South Wales, Sydney, Australia. From 1985 to 1987, he was with the Department of Industrial and Systems Engineering, National University of Singapore. In 1988, he returned to Australia as an Associate Professor with the Department of Mathematics, The University of Western Australia, Perth, Australia. In 1996, he joined, as a Professor, the Department of Mathematics and Statistics, Curtin University of Technology, Perth, where he is currently a Professor of applied mathematics and the Head of the Department of Mathematics and Statistics. From 1999 to 2004, he was the Chair Professor of Applied Mathematics and the Head of the Department of Applied Mathematics, The Hong Kong Polytechnic University, Kowloon, Hong Kong. He has delivered several keynote and fully funded invited lectures and has published five books, over 250 journal papers, and a number of conference papers. The software package MISER3.3, which is used for solving general constrained optimal control problems, was developed by the research team under his leadership. He is the Editor-in-Chief of the Journal of Industrial and Management Optimization. He also serves as an Associate Editor of a number of international journals, including Automatica, Nonlinear Dynamics and Systems Theory, the Journal of Global Optimization, Discrete and Continuous Dynamic Systems (Series A and Series B), and Dynamics of Continuous, Discrete and Impulsive Systems (Series A and Series $B$ ). He has also edited special issues for several journals, including Annals of Operations Research, the Journal of Global Optimization, and Dynamics of Continuous, Discrete and Impulsive Systems (Series A and Series B). His research interests include the theoretical and practical aspects of optimal control and optimization and their practical applications, such as in signal processing, telecommunications, and financial portfolio optimization. 